\title{
Упругие свойства графеноподобных соединений: модели Китинга и Харрисона
}

\author{
(C) С.Ю. Давыдов ${ }^{1}$, О.В. Посредник ${ }^{2}$ \\ ${ }^{1}$ Физико-технический институт им. А.Ф. Иофрфе РАН, \\ Санкт-Петербург, Россия \\ ${ }^{2}$ Санкт-Петербургский государственный электротехнический университет (ЛЭТИ), \\ Санкт-Петербург, Россия \\ E-mail: Sergei_Davydov@mail.ru
}

Поступила в Редакцию 16 октября 2020 г.

В окончательной редакции 16 октября 2020 г.

Принята к публикации 17 октября 2020 г.

Для 9 соединений IV-IV и 13 соединений III-V с использованием моделей Китинга и Харрисона получены оценки упругих постоянных третьего порядка, зависимости упругих постоянных второго порядка от давления, параметров Клейнмана и Грюнайзена и коэффициентов теплового расширения. Анализируются зависимость результатов от ионности связей по Филлипсу и расстояния между ближайшими соседями.

Ключевые слова: упругие постоянные, параметры Клейнмана и Грюнайзена, коэффициент теплового расширения.

DOI: 10.21883/FTT.2021.02.50487.221

1. В работе [1] Китинг предложил модель, содержащую силовые константы центрального взаимодействия ближайших соседей $\alpha$ и нецентрального взаимодействия вторых соседей $\beta$, для описания упругих постоянных второго порядка $C_{i j}$ гомополярных тетраэдрических кристаллов. В [2] Мартин обобщил модель Китинга на тетраэдрические гетерополярные соединения, учтя межионное кулоновское взаимодействие. В результате удалось вполне удовлетворительно описать упругие свойства кубических монокристаллов $\mathrm{A}_{\mathrm{N}} \mathrm{B}_{8-\mathrm{N}}[3]$.

Другим популярным подходом к описанию упругости соединений $\mathrm{A}_{\mathrm{N}} \mathrm{B}_{8-\mathrm{N}}$ является модель связывающих орбиталей (МСО) Харрисона [4], являющаяся упрощенным вариантом метода ЛКАО. В рамках $\mathrm{MCO}$ для $C_{i j}$ удалось получить аналитические выражения, содержащие константы центрального $k_{0}$ и нецентрального $k_{1}$ взаимодействий. Если в модели Китинга (как и в любой модели силового поля) константы $\alpha$ и $\beta$ определяются из экспериментальных данных по $C_{i j}$, то в модели Харрисона константы $k_{0}$ и $k_{1}$ вычисляются по известным значениям расстояний между ближайшими соседями (б.с.) и энергиям $s$ - и $p$-состояний атомов А и В кристалла. В настоящей работе мы рассмотрим упругие свойства соединений с двумерной гексагональной структурой, т.е. графеноподобные соединения (ГПС), используя оба подхода. Отметим, что, несмотря на более чем десятилетие исследований (в подавляющем большинстве, теоретических), интерес к механическим свойствам двумерных (2D) структур не затухает (см., например, [5-7]).

2. В работах $[8,9]$ модель Китинга была впервые применена к описанию гармонических и ангармонических свойств графена. В [8] было показано, что однослойной гексагональной гомополярной структуре отвечают упругие постоянные второго порядка $c_{i j}$ вида

$$
\begin{aligned}
& c_{11}=\frac{1}{\sqrt{3}}\left(4 \alpha+\beta+18 \frac{\alpha \beta}{4 \alpha+\beta}\right), \\
& c_{12}=\frac{1}{\sqrt{3}}\left(4 \alpha+\beta-18 \frac{\alpha \beta}{4 \alpha+\beta}\right),
\end{aligned}
$$

и параметр внутренних смещений Клейнмана $\xi=(2 \alpha-\beta) /(4 \alpha+\beta)$.

Недавно было убедительно продемонстрировано $[10,11]$, что у двумерных полярных соединений отсутствует щель между длинноволновыми продольными $\omega_{L O}(\Gamma)$ и поперечными $\omega_{T O}(\Gamma)$ оптическими фононами. Отсюда следует, что эффективный заряд ионов $Z^{*} \propto \sqrt{\omega_{L O}^{2}(\Gamma)-\omega_{T O}^{2}(\Gamma)}$ (поперечный динамический заряд Борна $[2,4])$ обращается в нуль. Таким образом, полученные в $[8,9]$ формулы применимы ко всем бинарным ГПС.

Результаты расчетов силовых константы $\alpha$, отношений $\beta / \alpha$ и параметров Клейнмана $\zeta$ приведены в таблице. Расчеты ионности связи по Филлипсу $f_{i}=1-\alpha_{c}^{3}$, где $\alpha_{c}$ - ковалентность связи по Харрисону [12-14], проводились по схеме работ [12-14] с использованием таблиц атомных термов Манна [15]. Расстояния между ближайшими соседями и значения модулей Юнга и коэффициентов Пуассона взяты из расчетов [16]. Для графена приведены результаты работ $[17,18]$. Из таблицы следует, что с ростом $f_{i}$ от 0 до 0.61 имеет место тенденция к уменьшению отношения $\beta / \alpha$, но не столь явная как для объемных соединений $\mathrm{A}_{\mathrm{N}} \mathrm{B}_{8-\mathrm{N}}[3,4,19]$. Более того, для соединений бора имеет место рост $\beta / \alpha$ 
с увеличением $f_{i}$. Значения $\xi$ с ростом $f_{i}$ немного увеличиваются. Отметим, что теоретически $\zeta$ может изменяться только в интервале от 0.2 при $\beta / \alpha=1$ до 0.5 при $\beta / \alpha=0$.

Так как в МСО ковалентная энергия связи $V_{2} \propto a^{-2}$, где $\alpha$ - расстояние между б. с., то, пренебрегая полярностью и металличностью связи, получим, что центральная силовая константа $k_{0}=\partial^{2} V_{2} / \partial a^{2} \propto^{-4}[12,13,18]$. На рисунке представлена зависимость отношения центральных силовых констант $\alpha / \alpha_{\mathrm{Gr}}$ ГПС от параметра $\left(a_{\mathrm{Gr}} / a\right)^{4}$, демонстрирующая, что $\alpha / \alpha_{\mathrm{Gr}} \sim\left(a_{\mathrm{Gr}} / a\right)^{4}$. Таким образом, между моделями Китинга и Харрисона имеется соответствие. Что же касается относительной роли нецентрального взаимодействия, определяемой в моделях Китинга и Харрисона соответственно отношениями $\beta / \alpha$ и $k_{1} / k_{0}$, то здесь ситуация иная: значения $\beta / \alpha$ существенно меняются в ряду ГПС, тогда как (в пренебрежении металличностью) значения $k_{1} / k_{0}$ остаются постоянными и равными $0.22[12,13,18]$. В этом состоит основное различие рассматриваемых моделей: по Китингу нецентральное взаимодействие ассоциируется с изменением угла между связями [1], центрированными на одном и том же атоме, а в МСО рассматривается взаимная разориентация образующих $\sigma$-связи гибридизованных орбиталей, принадлежащих соседним атомам [4].

3. Перейдем к оценкам ангармонических характеристик ГПС. Для упругих постоянных третьего порядка $c_{i j k}$ в работе [9] получены следующие выражения:

$$
\begin{gathered}
c_{11}=(16 \gamma / \sqrt{3}) \cdot\left[(1.5-\zeta)^{3}+4 \xi^{3}\right], \\
c_{222}=(16 \gamma / \sqrt{3}) \cdot\left[(0.5+\xi)^{3}+4(1-\xi)^{3}\right], \\
c_{112}=(16 \gamma / 3 \sqrt{3}) \cdot\left[(1.5-\zeta)^{2}(0.5+\zeta)+4 \xi^{2}(1-\zeta)\right],
\end{gathered}
$$

где $\gamma$ - ангармоническая силовая константа центрального взаимодействия ближайших соседей. При этом отправной точкой служила работа Китинга [20], где, однако, для описания $c_{i j k}$ дополнительно введены две нецентральные ангармонические константы. В работе [13] с использованием результатов расчетов [21,22] для графена получено значение $\gamma_{\mathrm{Gr}}=129 \mathrm{~N} / \mathrm{m}$. По аналогии с МСО, в рамках которой $\gamma \propto a\left(\partial^{3} V_{2} / \partial a^{3}\right) \propto a^{-4}$, положим $\gamma=\gamma_{\mathrm{Gr}}\left(a_{\mathrm{Gr}} / a\right)^{4}$. Таким образом, рисунок демонстрирует также зависимость отношения $\gamma / \gamma_{\mathrm{Gr}}$ от $\left(a_{\mathrm{Gr}} / a\right)^{4}$.

Оценки $\gamma$ и $c_{i j k}$ представлены в таблице. Какой-либо четкой зависимости $c_{i j k}$ от $f_{i}$ для рассмотренного ряда ГПС не наблюдается. Существуют, однако, характерные группы ГПС. Так, для соединений IV-IV наибольшие значения $c_{i j k}$ имеют $\mathrm{Gr}, \mathrm{SiC}, \mathrm{GeC}, \mathrm{SnC}$; для соединений III-V это BN, AlN, BP, GaN, причем максимальными значениями $c_{i j k}$ обладает графен. В целом, из таблицы следует, что $c_{111} \sim c_{222},\left(c_{111}+c_{222}\right) / 2 c_{112} \sim 5$.4. Некоторым исключением являются $\mathrm{GaAs}, \mathrm{InSb}$ и $\mathrm{AlSb}$, для которых $\left(c_{111}+c_{222}\right) / 2 c_{112} \sim 6.4$.

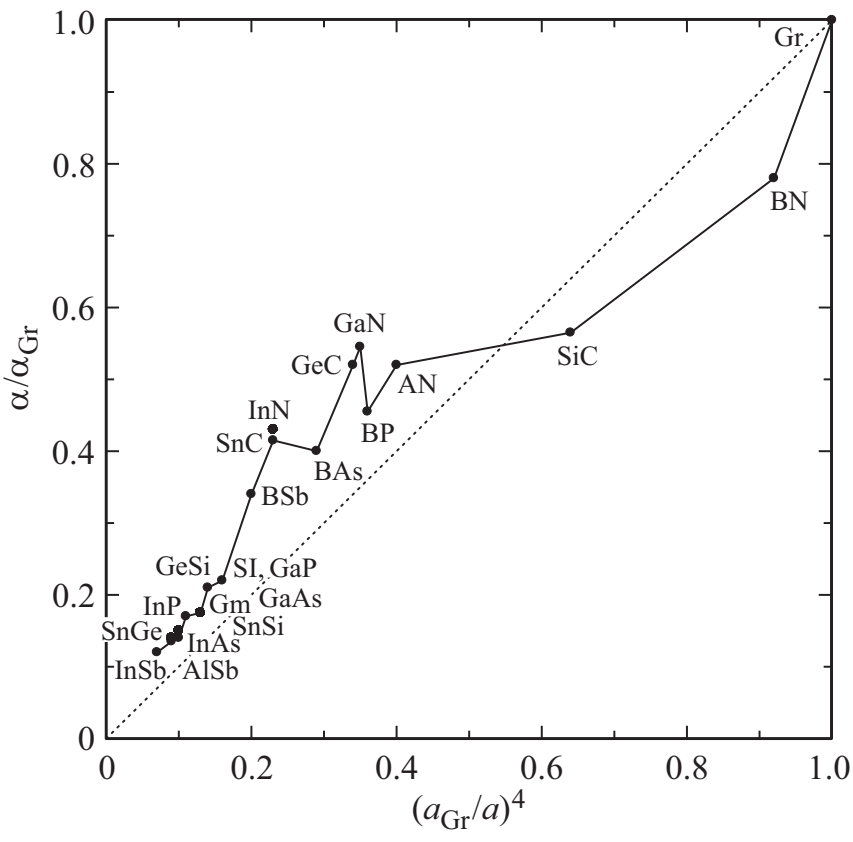

Зависимость отношения центральных силовых констант $\alpha / \alpha_{\mathrm{Gr}}$ модели китинга от параметра $\left(a_{\mathrm{Gr}} / a\right)^{4}(\mathrm{Gr}-$ графен, SI силицен, $\mathrm{Gm}$ - германен). Пунктирная линия отвечает равенству $\alpha / \alpha_{\mathrm{Gr}}=\left(a_{\mathrm{Gr}} / a\right)^{4}$.

Следующей упругой ангармонической характеристикой является зависимость упругих постоянных второго порядка $\tilde{c}_{i j}$ от двумерного давления $P[9]$ :

$$
\begin{gathered}
\tilde{c}_{11}=c_{11}-\left(c_{111}+c_{112}\right) \frac{1-\sigma}{E} P, \tilde{c}_{22}=c_{11}-c_{222} \frac{1-\sigma}{E} P, \\
\tilde{c}_{12}=c_{12}-c_{112} \frac{1-\sigma}{E} P .
\end{gathered}
$$

Результаты расчета параметров $\eta_{i j}=\partial \tilde{c}_{i j}(P) / \partial P$ представлены в таблице. Здесь также можно выделить характерные группы ГПС. Для соединений IV-IV наибольшие значения $\eta_{i j}$ имеют $\mathrm{SiC}, \mathrm{Gr}, \mathrm{SnSi}, \mathrm{Gm}$; для соединений III-V это BN, BP, AlN, GaP, причем максимальными значениями $\eta_{i j}$ обладает гексагональный нитрид бора. Из таблицы следует, что $\eta_{11} / \eta_{22} \sim 2, \eta_{11} / \eta_{12} \sim 11$.

Как показано в [18], имеет место соотношение

$$
\frac{\partial \tilde{B}}{\partial P}=-\frac{\eta_{11}+2 \eta_{12}}{3}=2 \bar{\gamma},
$$

где $\tilde{B}=\left(\tilde{c}_{11}+2 \tilde{c}_{12}\right) / 3$ - модуль всестороннего сжатия, $\bar{\gamma}$ - постоянная Грюнайзена. Значения $\bar{\gamma}$ приведены в таблице, откуда следует, максимальные постоянными Грюнайзена обладают $\mathrm{SiC}, \mathrm{BN}$ и $\mathrm{Gr}$. Численные расчеты [23] для графена дают $\bar{\gamma} \sim 2$, тот же результат получен в [18] в модели Харрисона. Какой-либо определенной зависимости $\bar{\gamma}$ от $f_{i}$ не наблюдается. То же относиться и к зависимости от $a$. 
Силовые константы $\alpha, \beta, \gamma$ (в ед. $\mathrm{N} / \mathrm{m})$, параметр внутренних смещений Клейнмана $\xi$, упругие постоянные третьего порядка $c_{i j k}$ (в ед. $\mathrm{N} / \mathrm{m})$, зависимости упругих постоянных второго порядка от давления $\eta_{i j}$, постоянные Грюнайзена $\bar{\gamma}$, отношения коэффициентов теплового расширения $\alpha_{T} / \alpha_{T}^{\mathrm{Gr}}$ и ионности связей $f_{i}$ по Филлипсу ( $\mathrm{Gr}-$ графен, $\mathrm{SI}-$ силицен, Gm - германен)

\begin{tabular}{|c|c|c|c|c|c|c|c|c|c|c|c|}
\hline $\mathrm{A}_{4} \mathrm{~B}_{4}$ & $\mathrm{Gr}$ & SI & $\mathrm{Gm}$ & $\mathrm{GeSi}$ & $\mathrm{SnSi}$ & $\mathrm{SnGe}$ & $\mathrm{SiC}$ & $\mathrm{GeC}$ & $\mathrm{SnC}$ & $\mathrm{BSb}$ & BAs \\
\hline$\alpha$ & 77 & 17.0 & 14 & 16 & 11.5 & 10.9 & 44 & 40 & 32 & 26 & 31 \\
\hline$\beta / \alpha$ & 0.90 & 0.56 & 0.59 & 0.59 & 0.61 & 0.50 & 0.67 & 0.59 & 0.47 & 0.58 & 0.68 \\
\hline$\xi$ & 0.22 & 0.32 & 0.31 & 0.31 & 0.30 & 0.33 & 0.28 & 0.31 & 0.34 & 0.31 & 0.28 \\
\hline$-\gamma$ & 129 & 18 & 17 & 18 & 13 & 12 & 63 & 44 & 30 & 26 & 37 \\
\hline $\begin{array}{l}-c_{111} \\
-c_{222} \\
-c_{112}\end{array}$ & $\begin{array}{c}2731 \\
2855 \\
517\end{array}$ & $\begin{array}{c}382 \\
400 \\
72\end{array}$ & $\begin{array}{c}355 \\
371 \\
67\end{array}$ & $\begin{array}{c}382 \\
400 \\
72\end{array}$ & $\begin{array}{c}273 \\
386 \\
52\end{array}$ & $\begin{array}{c}246 \\
257 \\
47\end{array}$ & $\begin{array}{c}1748 \\
1827 \\
331\end{array}$ & $\begin{array}{l}929 \\
971 \\
176\end{array}$ & $\begin{array}{l}628 \\
657 \\
119\end{array}$ & $\begin{array}{l}546 \\
571 \\
103\end{array}$ & $\begin{array}{l}792 \\
828 \\
150\end{array}$ \\
\hline $\begin{array}{l}-\eta_{11} \\
-\eta_{22} \\
-\eta_{12}\end{array}$ & $\begin{array}{l}14.0 \\
7.16 \\
1.30\end{array}$ & $\begin{array}{l}9.33 \\
4.77 \\
0.86\end{array}$ & $\begin{array}{l}10.1 \\
5.18 \\
0.94\end{array}$ & $\begin{array}{l}9.33 \\
4.77 \\
0.86\end{array}$ & $\begin{array}{l}11.4 \\
6.67 \\
0.90\end{array}$ & $\begin{array}{l}8.91 \\
4.55 \\
0.83\end{array}$ & $\begin{array}{l}15.3 \\
7.81 \\
1.42\end{array}$ & $\begin{array}{l}8.96 \\
4.58 \\
0.83\end{array}$ & $\begin{array}{l}7.74 \\
3.96 \\
0.72\end{array}$ & $\begin{array}{l}8.10 \\
4.14 \\
0.74\end{array}$ & $\begin{array}{l}9.67 \\
4.95 \\
0.99\end{array}$ \\
\hline $\bar{\gamma}$ & 2.77 & 1.84 & 2.00 & 1.79 & 2.01 & 1.76 & 3.02 & 1.76 & 1.53 & 1.60 & 1.94 \\
\hline$\alpha_{T} / \alpha_{T}^{\mathrm{Gr}}$ & 1 & 1.66 & 2.00 & 1.73 & 2.29 & 2.12 & 1.36 & 1.09 & 1.15 & 1.29 & 1.30 \\
\hline$f_{i}$ & 0 & 0 & 0 & 0 & 0.03 & 0.03 & 0.09 & 0.09 & 0.22 & 0.01 & 0.03 \\
\hline $\mathrm{A}_{4} \mathrm{~B}_{4}$ & $\mathrm{BP}$ & $\mathrm{BN}$ & $\mathrm{AlSb}$ & GaAs & $\mathrm{GaP}$ & $\mathrm{InSb}$ & AlN & $\operatorname{InP}$ & InAs & $\mathrm{GaN}$ & $\mathrm{InN}$ \\
\hline$\alpha$ & 35 & 60 & 11 & 14 & 17 & 9 & 40 & 13 & 11 & 42 & 33 \\
\hline$\beta / \alpha$ & 0.66 & 0.87 & 0.52 & 0.57 & 0.56 & 0.44 & 0.45 & 0.46 & 0.45 & 0.38 & 0.26 \\
\hline$\xi$ & 0.29 & 0.23 & 0.33 & 0.31 & 0.32 & 0.35 & 0.35 & 0.35 & 0.35 & 0.36 & 0.40 \\
\hline$-\gamma$ & 46 & 119 & 12 & 17 & 21 & 9 & 52 & 14 & 13 & 45 & 30 \\
\hline $\begin{array}{l}-c_{111} \\
-c_{222} \\
-c_{112}\end{array}$ & $\begin{array}{c}983 \\
1027 \\
186\end{array}$ & $\begin{array}{c}2513 \\
2627 \\
476\end{array}$ & $\begin{array}{c}246 \\
257 \\
47\end{array}$ & $\begin{array}{c}355 \\
371 \\
67\end{array}$ & $\begin{array}{c}437 \\
457 \\
83\end{array}$ & $\begin{array}{c}191 \\
200 \\
36\end{array}$ & $\begin{array}{c}1092 \\
1142 \\
207\end{array}$ & $\begin{array}{c}300 \\
314 \\
57\end{array}$ & $\begin{array}{c}273 \\
386 \\
52\end{array}$ & $\begin{array}{l}956 \\
999 \\
181\end{array}$ & $\begin{array}{l}628 \\
657 \\
119\end{array}$ \\
\hline $\begin{array}{l}-\eta_{11} \\
-\eta_{22} \\
-\eta_{12}\end{array}$ & $\begin{array}{l}10.7 \\
5.48 \\
0.99\end{array}$ & $\begin{array}{l}15.2 \\
7.77 \\
1.41\end{array}$ & $\begin{array}{l}9.05 \\
4.63 \\
0.85\end{array}$ & $\begin{array}{l}9.83 \\
5.02 \\
0.91\end{array}$ & $\begin{array}{l}9.85 \\
5.03 \\
0.91\end{array}$ & $\begin{array}{l}8.25 \\
4.22 \\
0.76\end{array}$ & $\begin{array}{l}10.4 \\
5.32 \\
0.96\end{array}$ & $\begin{array}{l}8.97 \\
4.59 \\
0.83\end{array}$ & $\begin{array}{l}11.4 \\
6.67 \\
0.90\end{array}$ & $\begin{array}{l}9.24 \\
4.72 \\
0.86\end{array}$ & $\begin{array}{l}7.86 \\
4.83 \\
0.73\end{array}$ \\
\hline $\bar{\gamma}$ & 2.11 & 3.00 & 1.79 & 1.94 & 1.95 & 1.63 & 2.05 & 2.01 & 2.20 & 1.83 & 1.55 \\
\hline$\alpha_{T} / \alpha_{T}^{\mathrm{Gr}}$ & 1.27 & 1.13 & 2.15 & 1.94 & 1.76 & 2.22 & 1.17 & 1.93 & 2.51 & 1.12 & 1.17 \\
\hline$f_{i}$ & 0.06 & 0.14 & 0.24 & 0.27 & 0.30 & 0.35 & 0.43 & 0.45 & 0.45 & 0.45 & 0.61 \\
\hline
\end{tabular}

В высокотемпературном пределе, когда температура значительно превышает температуру Дебая, коэффициент линейного расширения равен

$$
\alpha_{T}=2 k_{B} \frac{\bar{\gamma}}{a^{2} k_{0}},
$$

где $k_{B}-$ постоянная Больцмана, или $\alpha_{T} \propto \bar{\gamma} a^{2}$ [18]. Вводя отношения $\alpha_{T} / \alpha_{T}^{\mathrm{Gr}}=\left(\bar{\gamma} / \bar{\gamma}_{\mathrm{Gr}}\right)\left(a / a_{\mathrm{Gr}}\right)^{2}$, получим результаты, приведенные в таблице. Наибольшие значения этого отношения соответствуют InAs, $\mathrm{SnSi}$, $\mathrm{InSb}, \mathrm{AlSb}$ и $\mathrm{SnGe}$. Отметим, что в [24] для графена методом молекулярной динамики получено значения $\alpha_{T}^{\mathrm{Gr}}=6.5 \cdot 10^{-6} \mathrm{~K}^{-1}$, модель Харрисона дает $\alpha_{T} \approx 8.1 \cdot 10^{-6} \mathrm{~K}^{-1}[18]$.
4. Итак, в работе для двадцати двух ГПС приведены оценки упругих постоянных третьего порядка и зависимостей упругих постоянных второго порядка от давления, сделанные в рамках модели Китинга. Для определения силовых констант модель Китинга, как и все другие модели силового поля, требует привлечения экспериментальных данных или, по крайней мере, результатов расчетов из первых принципов. Так как среди всех ГПС такие данные имеются (насколько нам известно) только для графена, для нахождения гармонических $\alpha$ и ангармонических $\gamma$ констант центрального взаимодействия пришлось, основываясь на модели Харрисона, прибегать к скэйлингу, а именно полагать, что $\alpha / \alpha_{\mathrm{Gr}} \propto \gamma / \gamma_{\mathrm{Gr}} \propto\left(a_{\mathrm{Gr}} / a\right)^{4}$. Такой подход позволил про- 
стым способом описать упругость достаточно длинного ряда ГПС.

Зная упругие постоянные $c_{i j}$, легко определить характерные скорости звука в ГПС [25]. Продольная скорость звука $V_{p}=\sqrt{E(1-\sigma) / \rho_{c t l l}(1+\sigma)(1-2 \sigma)}$, сдвиговая, или поперечная скорость звука $V_{s}=\sqrt{c_{11} / \rho_{1}}$, где $E=\left(c_{11}^{2}-c_{12}^{2}\right) / c_{11}-$ модуль Юнга, $\sigma=c_{12} / c_{11}-$ коэффициент Пуассона и $\rho_{\text {cell }}=2 m_{\text {cell }} / 3 \sqrt{3} a^{2}-$ удельная плотность на элементарную ячейку $\left(m_{\text {cell }}-\right.$ суммарная масса атомов в элементарной ячейке). Воспользовавшись выражениями (3), можно определить зависимость скоростей звука от давления.

\section{Конфликт интересов}

Авторы заявляют об отсутствии конфликта интересов.

\section{Список литературы}

[1] P.N. Keating. Phys. Rev. 145, 637 (1966).

[3] R.M. Martin. Phys. Rev. B 1, 4005 (1970).

[3] С.П. Никаноров, Б.К. Кардашов. Упругость и дислокационная неупругость кристаллов. Наука, М. (1985).

[4] У. Харрисон. Электронная структура и свойства твердых тел. Мир, М.( 1983).

[5] K. Choudhary, G. Cheon, E. Reed, F. Tavazza. Phys. Rev. B 98, 014107 (2018).

[6] K.K. Al-Quraishi, Q. He, W. Kauppila, M. Wang, Y. Yang. Int. J. Smart Nano Mater. https://doi.org/10.1080/19475411.2020.1791276.

[7] H. Mei, Y. Zhong, D. He, X. Du, C. Li, N. Cheng. Sci. Rep. 10, 7873 (2020). https://doi.org/10.1038/s41598-020-64866-9.

[8] С.Ю. Давыдов. ФТТ 52, 756 (2010).

[9] С.Ю. Давыдов. ФТТ 53, 617 (2011).

[10] T. Sohier, M. Gibertini, M. Calandra, F. Mauri, N. Marzari. Nano Lett. 17, 3758 (2017).

[11] M. De Luca, X. Cartoixà, D.I. Indolese, J. Martín-Sánchez, K. Watanabe, T. Taniguchi, C. Schönenberger, R. Trotta, R. Rurali, I. Zardo. 2D-Materials 7, 035017 (2020).

[12] С.Ю. Давыдов. ФТП 47, 1065 (2013).

[13] С.Ю. Давыдов. Письма в ЖТФ 43, 5, 53 (2017).

[14] W.A. Harrison. Phys. Rev. B 27, 3592 (1983).

[15] W.A. Harrison. Phys. Rev. B 31, 2121 (1985).

[16] H. Sahin, S. Cahangirov, M. Topsakal, E. Bekaroglu, E. Akturk, R.T. Senger, S. Ciraci. Phys. Rev. B 80, 155453 (2009).

[17] C. Lee, X. Wei, J.W. Kysar, J. Hone. Science 321, 385 (2008).

[18] С.Ю. Давыдов, О.В. Посредник. ФТТ 57, 819 (2015).

[19] J.C. Phillips. Rev. Mod. Phys. 42, 317 (1970).

[20] P.N. Keating. Phys. Rev. 149, 674 (1966).

[21] E. Cadelano, P.L. Palla, S. Giordano, L. Colombo. Phys. Rev. Lett. 102, 235502 (2009).

[22] R. Wang, S. Wang, X. Wu, X. Liang. Physica B 405, 3501 (2010).

[23] N. Mounet, N. Marzari. Phys. Rev. B 71, 205214 (2005).

[24] M. Pazzo, D. Alfe, P. Lacovig, P. Hofmann, S. Lizzit, A. Baraldi. Phys. Rev. Lett. 106, 135501 (2011).

[25] S. Thomas, K.M. Ajith, S.U. Lee, M.C. Valsakumar. Roy. Soc. Chem. Adv. 8, 27283 (2018).

Редактор Т.Н. Василевскал 